\title{
Determinación del índice de generación y composición de residuos sólidos en la zona urbana del cantón de Turrialba, Costa Rica
}

\section{Determination of generation index and composition of solid waste from urban area in Turrialba, Costa Rica}

Silvia Soto-Córdoba ${ }^{\text {, Julio González-Buitrago² }}$

Fecha de recepción: 24 de setiembre de 2018

Fecha de aceptación: 16 de diciembre de 2018

Soto-Códoba, S; González-Buitrago, J. Determinación del índice de generación y composición de residuos sólidos en la zona urbana del cantón de Turrialba, Costa Rica. Tecnología en Marcha. Vol. 32-3. Julio-Setiembre 2019. Pág. 106-117.

DOI: https://doi.org/10.18845/tm.v32i3.4500

1 Doctora en Ciencias Ambientales, Catedrática. Escuela de Química, Centro de Investigación en Protección Ambiental CIPA, Instituto Tecnológico de Costa Roca. Costa Rica. Correo electrónico: ssoto@tec.ac.cr. (iD) https://orcid.org/0000-0002-3550-1505

2 Licenciado en Manejo de Recursos Naturales, funcionario del Ministerio de Salud de Costa Rica. Programa de Regulación de la Salud. Turrialba. Costa Rica. Correos electrónicos: julio.gonzalez@misalud.go.cr / jgbuitrago79@gmail.com 


\title{
Palabras clave
}

Residuos sólidos municipales; residuos sólidos comerciales; caracterización de residuos sólidos; índice de generación de residuo sólidos.

\section{Resumen}

Se realizó una investigación durante seis meses, en la zona urbana del cantón de Turrialba, localizado en la provincia de Cartago, con el propósito de identificar la composición de los residuos sólidos y la generación per cápita de los mismos. La investigación se realizó en 174 viviendas y 45 comercios para lo que se utilizó la metodología de estudios de composición aprobada en el país.

Los residuos sólidos se separaron manualmente y se clasificaron en las siguientes categorías: residuos orgánicos, papel, cartón, polilaminados, metales, residuos peligrosos, vidrio y misceláneos. El índice de generación por habitante determinado en la zona fue de $(0,58 \pm 0,04)$ $\mathrm{Kg} /$ persona/día. Se encontró que el 22 \% de los residuos sólidos corresponden a materiales que pueden recuperarse, $37 \%$ biodegradables y un $41 \%$ que deben llevarse al relleno sanitario. El resultado de esta investigación podrá ser utilizado por la municipalidad para promover la reducción de materiales no recuperables y diseñar un sistema de tratamiento de los residuos sólidos integrado ya que el actual sitio de disposición es un botadero.

\section{Keywords}

Municipal solid waste; commerce solid waste; solid waste characterization; generation rate.

\begin{abstract}
A six-month research was to conducted in urban area of Turrialba cantón, located in the province of Cartago, aims at identifying waste identifying waste composition and generation per capita. The investigation was carried out in 174 houses and using the national measure composition studies method.

Hand sorting was used, for classifying the waste into the next categories: organic wastes, paper, plastic, cardboard, polylaminates, metals, hazardous waste, glass and miscellaneous. The results for residential waste shows a solid waste generation of $(0,58 \pm 0,04) \mathrm{kg} / \mathrm{person} / \mathrm{day}$. There were found a $22 \%$ recoverable materials, $37 \%$ biodegradable materials and material for landfill disposition of $41 \%$. The results of this survey could be utilized for the municipality to promote the reduction of non-recoverable materials and design an integrated waste treatment, because the actual site for the disposition is a huge dumping.
\end{abstract}

\section{Introducción}

El diseño de un exitoso plan de manejo de residuos sólidos requiere información fidedigna de la cantidad y tipo de materiales que son generados en la comunidad, con el objetivo de planificar las rutas, tiempos de trabajo, potenciales de recuperación de materiales y estimar las propiedades de los Residuos Sólidos Municipales (RSM) según la ley respectiva de cada país. En Costa Rica, desafortunadamente, aún se siguen disponiendo en la corriente de los RSM algunos materiales peligrosos tales como baterías, jeringas desechables, disolventes y otros menores. Algunos estudios de composición que se han desarrollado a la fecha [1] [2] [3] [4] [5] [6] [7], se ha determinado la generación de RSM entre 0,7 y 0,4 kg/persona/día. De acuerdo con el ordenamiento legal del país, las municipalidades están obligadas a manejar los residuos sólidos y a desarrollar planes de accion donde se incluye la realización de estudios composición y caracterización, así como determinación del indice de generación por habitante. 
Esta investigación se realizó en el cantón de Turrialba que es el quinto de la provincia de Cartago, esta localizado entre 090 47, 14 < latitud norte y 830 34, 03» longitud Este, tiene 49,61 $\mathrm{Km}^{2}$ y una población estimada de 69615 habitantes [8].

El Cantón de Turrialba posee 20453 viviendas ocupadas, con un promedio de 3,4 habitantes por unidad habitacional. Está dividido en doce distritos Chirripó, Tuis, Tayutic, La Suiza, Turrialba, La Isabel, Santa Rosa, Santa Cruz, Peralta, Santa Teresita, Pavones y Tres Equis. De los cuales las reservas indígenas representan un 65\% del territorio del cantón, y los porcentajes de población urbana son de un 57\% contra un 42 \% de población rural. Es el sexto cantón nacional con menor crecimiento de población [8]. En las zonas rurales no existe recolección de RSM. La municipalidad solo atiende a los distritos de Turrialba y la Isabel, por su parte, las zonas indígenas, están prácticamente olvidadas de programas de saneamiento ambiental por parte de la municipalidad, aunque los habitantes de la zona se han organizado para gestionar sus residuos sólidos.

En el cantón de Turrialba, la primera investigación realizada para determinar los índices de generación y de composición fue realizada en el 2002 [9], según este estudio, la cantidad de RSM descargadas en el botadero de Turrialba ascendía a 46800 toneladas/año, sin embargo, este dato no corresponde con la realidad observada en el sitio de vertido. El segundo estudio relacionado con residuos sólidos fue desarrollado por estudiantes de la Universidad de Costa Rica, en el mismo se determinaron descargas de 65 Toneladas/día en el botadero de Turrialba (Escuela de Geográfia, 2009), desafortunadamente a la fecha no se disponen de registros del indice de generación por habitante. La incidencia de pobreza en el cantón aumenta desde los distritos más urbanos (Turrialba, Santa Rosa, Santa Cruz) con valores entre 23 y 38 \% de pobreza, hasta distritos extremadamente deprimidos y rurales como Tres Equis, Santa Teresita, Peralta, Tayutic, con porcentajes de pobreza superiores al $60 \%$ y valores de pobreza extrema superiores al $20 \%$ [10].

Los RSM son enviados directamente al botadero municipal de Turrialba que no cuenta con medidas de protección ambiental, restricción de ingreso de personas, es un foco de contaminación e incluso en algunas ocasiones se han presentado incendios. Se estima el ingreso anual de residuos sólidos en el orden de 10300 Toneladas métricas.

En Costa Rica a partir del año 2010 [11] se hace obligatorio el manejo adecuado de los RSM y en el año 2012 se publica el primer reglamento [12] [13] para realizar los respectivos estudios de composición, a la fecha es obligatorio para todos los cantones contar estos estudios de generación y composición de RS. El reglamento de estudios de composición tiende a establecer un método general para realizar los estudios de composición, sin embargo, los autores consideran que es necesario realizar una mejor y más robusta interpretación estadística de los resultados, a fin de poder estimar y predecir las cantidades máximas y mínimas generadas en los cantones.

El objetivo primario de este estudio fue desarrollar una estimación representativamente estadistica de la composición de los residuos sólidos urbanos del cantón de Turrialba, así como estimar la generación de RMM según el estrato socioeconomico.

\section{Métodos y Materiales}

\section{Área de estudio}

El estudio se realizó en la zona urbana del Cantón de Turrialba, distritos de Turrialba y la Isabel ( \#1 y \# 11) en las comunidades de: Turrialba centro, Calle Puntarenas, La Guaria, Los Ángeles, Recope, Las Américas, Noche Buena, Carmen Lyra, Olivos Nuevos, Azul. Se llevó a cabo durante seis meses en el año 2015. No se consideró la zona indígena ni áreas protegidas, también se excluyen zonas rurales. 
En la figura 1 se muestra un mapa desarrollado por el Ministerio de Hacienda, donde se observan las distribuciones de terrenos, según el valor de los mismos, en las zonas urbanas de la provincia de Turrialba, esta distribución de valores de propiedad se utilizó para clasificar los estratos socioeconómicos.

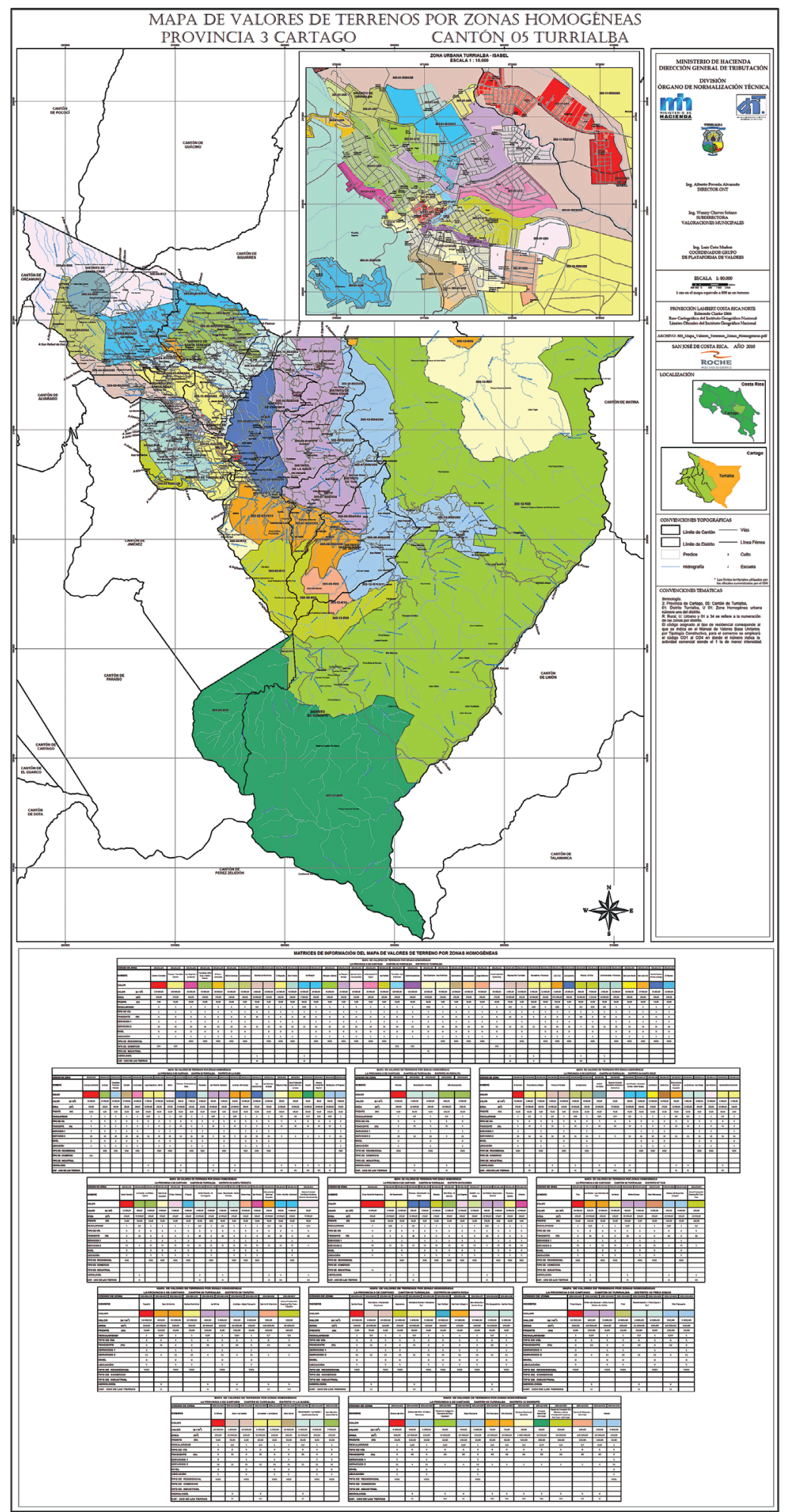

Figura 1. Mapa valores de terrenos del Cantón de Turrialba [14] 


\section{Estimación de la cantidad de RSM en el sector residencial}

La estimación de la cantidad de RSM (kg/persona/día), se realizó utilizando la "Metodología para estudios de generación y composición de residuos sólidos" [15]. Para adaptar el reglamento al área de estudio fue necesario realizar las siguientes presunciones:

1. La definición del estrato socioeconómico: En el país no se cuentan con estudios claros que definan los estratos socioeconómicos en una región, a menos se utilice el Índice de Desarrollo Social que está definido para los distritos, sin embargo, este dato no describe el detalle necesario para realizar la investigación, debido a que la zona de estudio correspondía al área donde la municipalidad recolecta los RSM y son dos distritos diferentes, fue necesario hacer uso del mapa de valores por zonas homogéneas desarrollado por el ministerio de Hacienda, para identificar los estratos socioeconómicos en los vecindarios estudiados.

2. La clasificación de los estratos socioeconómicos: La herramienta de estudios de composición propone el estudio en tres estratos: uno de ingresos altos, otro de ingresos medios y finalmente un estrato de bajos ingresos, pero no define los límites para clasificar dichos estratos. El único instrumento que se encontró para predecir el nivel socioeconómico fue el mapa del Ministerio de Hacienda [16] que agrupa 10 diferentes categorías de valores de terrenos. Luego de consultar con diferentes actores sociales de la provincia, se decidió construir la siguiente clasificación: Clase de mayores ingresos (Al), que poseen casas bien construidas, viven en terrenos más caros mayor de 50000 colones $/ \mathrm{m}^{2}$, Clase de ingresos medios (MI), sus ingresos son suficientes para tener un vehículo, casa, y viven en terrenos cuyo costo ronda entre 50000 y 25000 colones $/ \mathrm{m}^{2}$ y clase de ingresos bajos (BI), el costo del terreno donde viven es inferior a 25000 colones/ $\mathrm{m}^{2}$.

En el área de estudio hay 11976 viviendas distribuidas, de las cuales, según el mapa de valores del Ministerio de Hacienda y la clasificación previamente indicada, se logró categorizar según se muestra en el cuadro 1.

Cuadro 1. Distribución de las viviendas según estrato socioeconómico

\begin{tabular}{|c|c|c|}
\hline Estrato & Número de viviendas & Porcentaje por Estrato \\
\hline $\mathrm{Al}$ & 509 & $4,25 \%$ \\
\hline $\mathrm{MI}$ & 5861 & $48,94 \%$ \\
\hline $\mathrm{BI}$ & 5606 & $46,81 \%$ \\
\hline
\end{tabular}

Usando los datos del INEC para esta zona se observa un promedio de 3,4 de personas por vivienda, la población de la zona de estudio es 40718 habitantes, lo que representa un $58,5 \%$ de la población de todo el cantón, y a la vez corresponde a la población urbana del mismo.

Se asume para efectos de cuantificación que la distribución de viviendas en el cantón sigue el mismo patrón porcentual, aunque se sabe que el resto es principal.

3. La selección de las zonas de estudio: se consultó con los funcionarios del departamento municipal de patentes, el departamento de catastro, los conductores de los camiones recolectores de RS y se identificaron las zonas o barriadas donde se agrupaban las poblaciones que se podrían categorizar en cada uno de los estratos, por lo que se aprovechó esta característica para diseñar una estructura de muestreo en forma bloques geográficos, se muestran cuatro ejemplos de dichos bloques en la figura 2. 



Figura 2. Ejemplo de la sectorización de barrios para la realización de la investigación.

4. Número de viviendas encuestadas: Este número se calculó usando la ecuación 1.

$$
n=\frac{\left(z^{2} * N^{*} \delta^{2}\right)}{(N-1) * E^{2}+\left(Z^{2} * \delta^{2}\right)} * 1,25
$$

$n=$ número de viviendas encuestadas.

$N=$ Cantidad total de residencias en Turrialba.

$Z=$ coeficiente de confidencia al 95\%=1,96.

$\delta$ = Desviación estándar $=0,3 \mathrm{Kg} /$ persona/día, este dato es suministrado en el reglamento respectivo.

$E=$ Error $=0,05 \mathrm{Kg} /$ persona/día .

Se sumó un $25 \%$ de unidades habitacionales para compensar la no respuesta. En total se encuestaron 10 viviendas del tipo IA, 84 viviendas tipo MA, y 80 viviendas del tipo BA.

5. Para conocer con exactitud la cantidad de personas que habitan en cada casa se aplicó previo al estudio un cuestionario para conocer el número de personas que habita cada vivienda, además se aprovecha para explicar el proceso del estudio y coordinar fechas de recolección.

6. Las casas seleccionadas fueron marcadas con un identificador.

7. La generación per cápita diario (IG) en cada estrato socioeconómico fue determinado usando las ecuaciones 2, 3 y 4. 
$\mid \mathrm{G}_{\mathrm{Al}}=\frac{\mathrm{Kg} \text { de RSM recolectada / casa tipo AI }}{\text { Número total de personas en cada casa } \mathrm{x} \text { total de días de generación de RSM }}$

Kg de RSM recolectada / casa tipo MI

$\mathrm{IG}_{\mathrm{MI}}=\overline{\text { Número total de personas en cada casa x total de días de generación de RSM }}$

Kg de RSM recolectada / casa tipo BI

$\mathrm{IG}_{\mathrm{BI}}=\overline{\text { Número total de personas en cada casa } x \text { total de días de generación de RSM }}$

Posteriormente se realiza la sumatoria de los IG de cada estrato considerando su contribución porcentual de forma hasta determinar el Índice de generación residencial (IGresidencial).

8. La cantidad total de RSM que son enviados a vertedero en Turrialba se determinó considerando la población en cada estrato indicado en el cuadro 1 ,

$$
(I G A I \times P A I+\mid G M I \times P M I+I G B I \times P B I) \times \text { Pdistritos }=\text { GT }
$$

GT = Generación total del cantón

$P_{\text {distritos }}=$ Población total en la zona de estudio, 40718 habitantes.

$P_{A I}=$ Fracción porcentual de la población en estrato Al, corresponde a 4,25 \%

$P_{M I}=$ Fracción porcentual de la población en estrato MI, corresponde a 48,94\%

$\mathrm{P}_{\mathrm{BI}}=$ Fracción porcentual de la población en estrato BI, corresponde a 46,81 \%

Se realizó un análisis estadístico a los resultados de IG en cada estrato para establecer la normalidad de los datos usando como criterio el p-value de 0,05 caso contrario de no observar normalidad, se aplicaron transformaciones estadísticas para alcanzar la normalización. Posteriormente, conociendo la generación per cápita por día de cada estrato, se aplicó el test de ANOVA con un 95 \% de confianza para comparar los IG de cada estrato y determinan diferencias significativas.

\section{Recolección de la muestra y separación manual}

Aleatoriamente se seleccionaron las viviendas que se utilizaron en el estudio. Desde cada casa seleccionada se recolectaron las bolsas, cajas, contenedores o cualquier material donde se depositaban los RSM, se trasladaron a un pick up y se enviaron a un espacio destinado en el plantel municipal de Turrialba. Cada bolsa fue abierta y se verificó manualmente la existencia de cualquier material peligroso que se eliminó de la muestra, los residuos más voluminosos fueron reducidos de tamaño con ayuda de una tijera de podar y cuchillo de chapia, con el objetivo de realizar adecuadamente el cuarteo para la toma de muestra del estudio de composición.

Se colocaron cerca de $50 \mathrm{~kg}$ en el suelo. Se mezcló con palas y una vez lista se inició con el cuarteo [12], los colaboradores tenían medidas de seguridad y las vacunas respectivas para esta labor.

En atención al reglamento respectivo se determinaron los pesos respectivos de las 11 categorías de residuos sólidos a saber:

Biodegradables:

- Restos de comida, desechos de jardín, hojas, basura de pequeña agroindustria 
Reciclables:

- Papel (revistas, periódicos, libros, material de empaque, papel blanco)

- Cartón

- Plásticos (PET, HDPE, LDPE, PVC)

- Vidrio (excepción vidrio plano)

- Metales incluyendo Aluminio

- Polilaminados

Desechos peligrosos

- Desechos médicos, disolventes, baterías, plaguicidas, pinturas

No Reciclables

- Electrónicos menores

- Misceláneos (servilletas, toallas sanitarias, piedras, tierra, desechos de construcción y demolición, cuero, madera, gomas, cauchos, etc)

- Textiles

El porcentaje de composición de cada uno de los componentes en los RSM fue calculado usando las fórmulas 6, 7 y 8

Porcentaje de composición de la fracción de Al,

$$
\mathrm{PC}_{\mathrm{Al}}=\frac{\mathrm{Kg} \text { de los RSM de la fracción } \mathrm{AI}}{\text { Total Kg de la muestra }} \times 100
$$

Porcentaje de composición de la Fracción de MI,

$$
\mathrm{PC}_{\mathrm{MI}}=\frac{K g \text { de los } \mathrm{RSM} \text { de la fracción } \mathrm{MI}}{\text { Total } \mathrm{Kg} \text { de la muestra }} \times 100
$$

Porcentaje de composición de la Fracción de BI,

$$
\mathrm{PC}_{\mathrm{Al}}=\frac{\mathrm{Kg} \text { de los RSM de la fracción BI }}{\text { Total Kg de la muestra }} \times 100
$$

El cálculo total de generación según cada fracción se determinó usando la ecuación 9.

$$
\begin{gathered}
\left(P_{A l} \times P_{A l}+P C_{M l} \times P_{M l}+P_{B l} \times P_{B l}\right) \times P_{\text {distritos }} \times \mathrm{kg} \text { de la muestra= GFx } \\
G F x=\text { Generación según cada fracción. }
\end{gathered}
$$

\section{Sector Comercial}

En Turrialba y La Isabel existen 960 comercios, de los cuales se definió una muestra representativa de 45 comercios al azar, para esto se utilizó la fórmula 1, con una desviación estándar de 0,5 kg/habitante/día y un error permisible de 0,15 kg/habitante/día.

La generación de los comercios (IGcomercial) se calculó como se muestra en la fórmula 10. En Turrialba a la fecha del estudio existían 960 comercios.

$$
I G_{\text {Comercios }}=\frac{\text { Kg de RSM recolectados en los comercios }}{D R S M \times N \text { Comercios }} \times \frac{\text { Total de comercios }}{\text { Pdistritos }}
$$


$\mathrm{D}_{\mathrm{RSM}}=$ cantidad de días en que se recolectaron los RSM

$\mathrm{N}_{\text {comercios }}=$ Número de comercios en la muestra (45 comercios )

$\mathrm{T}_{\text {Comercios }}=$ Cantidad total de comercios en la zona de estudio (900 comercios)

$P_{\text {distritos }}=$ Población total en la zona de estudio, 40718 habitantes.

Calculo de la generación total en el cantón (IG)

Para determinar la generación total se debe sumar el IGcomercios y IGresidencial

$$
I G_{\text {total }}=I G_{\text {comercios }} \text { y } I G_{\text {residencial }}
$$

Análisis estadístico

La relación entre el índice de generación según cada estrato socioeconómico se ejecutó usando el estadístico p-value. Se utilizó el software Minitab 17.

\section{Resultados}

Estimación de la cantidad de RSM generados en el Cantón de Turrialba

Viviendas

El resultado del cálculo del índice de generación por habitante en el sector residencial para el estrato Al fue de 0,27 \pm 0,04 kg/día/habitante, la prueba estadística de comparación de grupos entre los estratos $\mathrm{Ml}$ y $\mathrm{Bl}$ no mostró diferencias significativas entre ambos por lo que se fundieron en un solo grupo con un índice de generación de 0,48 0,04 kg/día/habitante, el promedio respectivo para ambos estratos considerando sus contribuciones porcentuales fue de $0,471 \pm 0,04 \mathrm{~kg} /$ día/habitante.

\section{Comercios}

La cantidad de residuos recolectados en el área de estudio alcanzó los 2,84kg/comercio/día, en atención al número de comercios se estima una generación diaria de 2726 kg/día/comercio, para determinar el índice de generación por habitante se utilizó la fórmula 10, por lo que el indicador para este sector es de 0,067 kg comercio/persona/día.

Generación total en el Cantón

El IG del cantón calculado según la fórmula 11 es de 0,54 \pm 0,04 kg/persona/día, con lo cual se proyecta un promedio de Toneladas de RSM para la zona de estudio de 21,91 Toneladas/día y un gran promedio total de 8026 Toneladas al año. En el botadero de Turrialba se recolectan RSM provenientes del Cantón de Juan Viñas y Alvarado, además de otras contribuciones de agroindustrias, por esta razón los resultados de la investigación son congruentes con el valor reportado por ingreso en el botadero de Turrialba de 10300 Toneladas anuales. En el cuadro 2 se muestra un estimado según estratos considerando la contribución de los comercios para la generación máxima y mínima de RSM. 
Cuadro 2. Índices de generación residencial determinados para cada estrato socioeconómico y estimación de la cantidad enviada diariamente al vertedero de Turrialba en el área de estudio.

\begin{tabular}{|c|c|c|c|c|}
\hline Estrato & $\begin{array}{c}\text { Generación per cápita } \\
\text { (kg/día/habitante) }\end{array}$ & $\begin{array}{c}\text { Promedio Toneladas/ } \\
\text { día enviada al } \\
\text { vertedero }\end{array}$ & $\begin{array}{c}\text { Cantidad Mínima } \\
\text { en Toneladas/ } \\
\text { día enviada al } \\
\text { vertedero }\end{array}$ & $\begin{array}{c}\text { Cantidad Máxima } \\
\text { en Toneladas/ } \\
\text { día enviada al } \\
\text { vertedero }\end{array}$ \\
\hline $\mathrm{Al}$ & $0,34 \pm 0,04$ & 0,59 & 0,52 & 0,66 \\
\hline $\mathrm{Ml} \mathrm{y} \mathrm{Bl}$ & $0,55 \pm 0,04$ & 21,44 & 19,88 & 23,00 \\
\hline $\begin{array}{c}\text { Todos los } \\
\text { estratos }\end{array}$ & $0,54 \pm 0,04$ & 21,99 & 20,36 & 23,62 \\
\hline $\begin{array}{c}\text { Anuales } \\
\text { (Ton RSM/año) }\end{array}$ & & 8026 & 7431 & 8620 \\
\hline
\end{tabular}

\section{Composición de los residuos sólidos}

En el cuadro 3 se muestran los resultados del estudio de composición de las viviendas, los residuos biodegradables junto con otros residuos recuperables, los cuales alcanzan un 76\% de los RSM.

Cuadro 3. Proporción porcentual de los componentes encontrados en los RSM según estrato socioeconómico

\begin{tabular}{|c|c|c|c|c|}
\hline Categorías & $\begin{array}{c}\text { Estrato alto } \\
(4,54 \%)\end{array}$ & $\begin{array}{c}\text { Estrato medio } \\
(47,43 \%)\end{array}$ & $\begin{array}{c}\text { Estrato bajo } \\
(48,02 \%)\end{array}$ & $\begin{array}{c}\% \text { /Ponderado/ } \\
\text { Categoría }\end{array}$ \\
\hline Biodegradable & $27,9 \%$ & $45,88 \%$ & $30,15 \%$ & $37,51 \%$ \\
\hline Papel & $13,95 \%$ & $8,12 \%$ & $5,15 \%$ & $6,96 \%$ \\
\hline Cartón & 12,79 & $3,42 \%$ & $2,30 \%$ & $3,31 \%$ \\
\hline Plásticos & $13,95 \%$ & $8,12 \%$ & $10,31 \%$ & $9,44 \%$ \\
\hline Metales & - & $1,89 \%$ & $0,55 \%$ & $1,16 \%$ \\
\hline Textiles & $4,65 \%$ & - & $3,39 \%$ & $1,84 \%$ \\
\hline Polilaminados & $1,16 \%$ & $0,71 \%$ & $0,44 \%$ & $0,60 \%$ \\
\hline Peligrosos & - & $0,01 \%$ & - & $0,000 \%$ \\
\hline Vidrio & - & $0,88 \%$ & 0,60 & $0,71 \%$ \\
\hline Otros & $25,58 \%$ & $30,97 \%$ & $47,11 \%$ & $38,47 \%$ \\
\hline
\end{tabular}

En el caso del sector comercial la distribución de los componentes varía como se muestra en la figura 3. En este sector existen posibilidades de valorización del papel, cartón y plástico principalmente. 


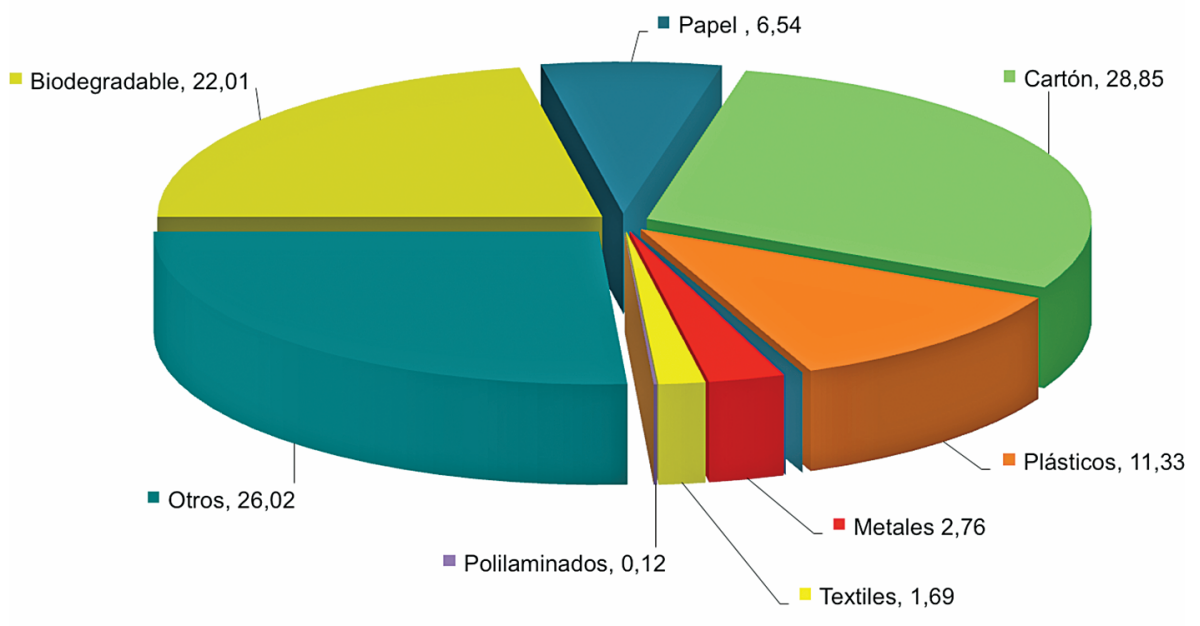

Figura 3. Distribución porcentual de los materiales desechados en los comercios.

\section{Discusión y conclusiones}

Este articulo muestra el primer estudio de generación y composición de RSM realizado en Turrialba.Los estudios de composición y la determinación de los índices de generación son herramientas útiles para el diseño de políticas específicas en el sector municipal, con esto se puede identificar las diferentes posibilidades valorización, establecer estrategias de educación ambiental dirigidas a la recuperación en la fuente según los estratos socioeconómicos, y adecuar los presupuestos para garantizar la sustentabilidad del servicio.

De acuerdo a los resultados de composición, el principal material desechado corresponde a los biodegradables con un $37,5 \%$, seguido por otros $38,5 \%$, dichos materiales son enviados a un relleno sanitario incontrolado y la municipalidad no tiene una visión del tratamiento adecuado de esta fracción. El papel y el cartón pueden sumarse a la contribución del material biodegradable incrementando la cantidad de residuos valorizables en un $48 \%$, estos materiales podrían utilizarse en procesos como compostaje, biogás y bioetanol.

Es notorio como a pesar de la percepción general de una mayor generación de RSM conforme aumenta el nivel socioeconómico, resulta que justo los sectores con mayores ingresos producen menos RSM, esto puede deberse en el caso estudiado precisamente al uso de restaurantes fuera de casa, menor tiempo en el hogar y producción de RSM con menor masa, llama la atención el menor porcentaje de residuos biodegradables y un aumento importante en desechos de papel, plásticos y textiles. La situación es inversa en los sectores con ingresos medios y bajos, ahí predominan la generación de materiales biodegradables, a su vez, conforme disminuye el poder adquisitivo, se observa menor producción de papel y cartón

El índice de generación en la zona alcanza $0,54 \mathrm{~kg} /$ persona/día, por lo que se prevé cerca de 22 toneladas diarias de residuos, de las cuales casi 9 toneladas corresponden a residuos biodegradables que podrían recuperarse en sistemas de reciclaje.

La municipalidad ocupa mejorar el actual botadero en el cual de disponen cerca de 10,300 toneladas de RSM al año, según los datos recopilados en campo, y que no cuenta con ningún control ambiental. Urge invertir en programas de educación ambiental para educar a la población en la correcta separación de residuos y con esto incrementar la separación de la basura en la fuente. 


\section{Referencias}

[1] R. Campos y S. Soto-Córdoba, Estudio de Generación y Composición en Guácimo, Guácimo, Limón, 2013.

[2] J. Herrera-Murillo, R.-M. J y D. Anchía-Leitón, «Generarion rates and characterization of ordinary solid waste in four municipalities of the metropolitan area Costa Rica,» Revista Geográfica de America Central, vol. 2, n 57, 2016.

[3] M. Sabillón, P. Umaña y S. Chacón, «Municipalidad de San Carlos,» [En línea]. Available: https:// www.munisc.go.cr/documentos/Secciones/18/Estudio\%20de\%20cuantificaci\%C3\%B3n\%20y\%20caracterizaci\%C3\%B3n\%20de\%20los\%20Residuos\%20S\%C3\%B3lidos\%20en\%20San\%20Carlos..pdf.

[4] S. Soto-Córdoba y O. Quijada, «Estudio de composición de los residuos sólidos urbanos en Esparza, Puntarenas, Costa Rica,» Tecnología en Marcha, pp. 23-32, 2008.

[5] K. Carranza-Campos y M. Monge-Leiva, «Análisis tecno-económico y ambiental de una tecnología de tratamiento térmico para la generación de energía eléctrica mediante los residuos sólidos urbanos de la zona de los santos,» 2014 febrero 2014. [En línea]. Available: http://repositorio.sibdi.ucr.ac.cr:8080/jspui/bitstream/123456789/2010/1/36118.pdf.

[6] Municipalidad de Alajuela, «Sitio Web Municipalidad de Alajuela,» 2013. [En línea]. Available: http:// www.munialajuela.go.cr/documentos/planificacion/INTEGRADO\%20DOCUMENTOS\%2OIMPORTANTES\%20 ALCALDIA-selected/PLAN\%20GESTION\%20DE\%20RESIDUOS/PlanMunicipal_GestionResiduos.pdf.

[7] S. Vallejo, «Diagnóstico de la gestión de los desechos sólidos generados en la comunidad de Guácimo de Limón para el cumplimiento de la ley 14423,» UCI, San José, 2011.

[8] INEC, «INEC Costa Rica,» 23 Febrero 2011. [En línea]. Available: http://www.inec.go.cr/Web/Home/ GeneradorPagina.aspx.

[9] Organización Panamericana de la Salud (OPS), «Evaluación Regional de los Servicios de Manejo de Residuos Sólidos EVAL - RS,» 2012.

[10] R. A. Ramírez, «Turrialba una economia local entre la crisis y el desarrollo,» Ciencias Económicas, pp. 2009231, 2009.

[11] Asamblea Legislativa, «Ley para la Gestión Integral de Residuos,» Costa Rica, 2010.

[12] Programa de Competitividad y Medio Ambiente (CYMA), "Guía de interpretacón de la metodología para la realización de estudios de generación y composición de residuos ordinarios,» Costa Rica, 2012.

[13] Programa Competitividad y Medio Ambiente (CYMA), «Manula para la Elaboración de Planes Municipales de Gestión Integral de Residuos Sólidos,» San José, 2008.

[14] Ministerio de Hacienda, «Mapas de valores de terrenos por zonas homogéneas,» Octubre 2013. [En línea]. Available: http://www.hacienda.go.cr/docs/545cdd5ca8e39_5.pdf.

[15] Gobierno de Costa Rica, Decreteo Ejecutivo 37745 del 19/4/2013, San José: La Gaceta, 2013. 